\title{
Diagnóstico de Falhas em Estruturas Mecânicas Utilizando uma Rede Neural Artificial ARTMAP-Fuzzy
}

\author{
$\underline{\text { Fernando P. A. Lima }}^{\dagger} \quad$ Adriano. S. Souza ${ }^{\dagger} \quad$ Simone. S. F. Souza \\ Mara. L. M. Lopes ${ }^{*} \quad$ Fábio R. Chavarette \\ ${ }^{\dagger}$ Departamento de Engenharia Mecânica, Faculdade de Engenharia de Ilha Solteira (FEIS) \\ *Departamento de Matemática, Faculdade de Engenharia de Ilha Solteira (FEIS) \\ "Departamento de Engenharia Elétrica, Faculdade de Engenharia de Ilha Solteira (FEIS) \\ Universidade Estadual Paulista "Júlio de Mesquita Filho" (UNESP), Ilha Solteira, SP, BRASIL \\ E-mails: engfernandoparra@gmail.com, eng.adrianosouza@gmail.com, \\ simonefrutuoso.mat@gmail.com, mara@mat.feis.unesp.br, fabioch@ mat.feis.unesp.br
}

\begin{abstract}
Palavras-chaves: Diagnóstico de Falhas Estruturais, Estruturas Mecânicas, Redes Neurais Artificiais, ARTMAP-Fuzzy.

Resumo: Este artigo apresenta uma rede neural artificial ARTMAP-Fuzzy para monitoramento e diagnóstico de falhas em estruturas mecânicas. Portanto, o objetivo é utilizar a rede neural ARTMAP-Fuzzy na identificação e caracterização de amostras de falhas estruturais. Esta metodologia pode auxiliar os profissionais na inspeção de estruturas mecânicas, visando identificar e caracterizar falhas, a fim de realizar manutenção preventiva e a tomada de decisões. Para validar a metodologia foi realizada a modelagem e simulação de sinais a partir de um modelo numérico utilizando uma viga de alumínio. Os resultados obtidos pela rede neural ARTMAP-Fuzzy apresentam eficiência, robustez e precisão.
\end{abstract}

\section{Introdução}

Cada vez mais a manutenção preventiva vem sendo utilizada no dia-a-dia das indústrias, empresas, construções, monitoramento de maquinas, entre outros. Sua utilização é justificada devido à necessidade de reduzir gastos e aumentar a confiabilidade e segurança das estruturas e equipamentos, evitando catástrofes a partir de falhas estruturais [5].

As falhas estruturais podem ocorrer devido a diversos fatores tais como: desgaste de um componente, afrouxamento de uniões aparafusadas, trincas ou mesmo a combinação destes elementos. Independente da origem e intensidade, na grande maioria dos casos, a falha estrutural provoca uma sensível variação dos parâmetros espaciais da estrutura tais como: redução da rigidez estrutural, ligeira redução da massa e um aumento do amortecimento, o que leva a uma mudança do comportamento dinâmico da estrutura. Assim, a variação dos parâmetros espaciais afeta os principais parâmetros dinâmicos, funções de respostas, frequências de ressonância, razão de amortecimento e modos próprios da estrutura [10].

Existem algumas técnicas de manutenção preventiva baseadas em ensaios não-destrutivos (NDE) que já são largamente aplicadas, dentre elas tem-se: análise de óleo, partículas magnéticas, líquidos penetrantes, métodos baseados na análise de vibrações, etc. A exigência cada vez maior dessas técnicas e sua utilização nas mais diversas situações, levam a constantes pesquisas envolvendo novos materiais e o desenvolvimento de novas técnicas para monitoramento e diagnóstico de falhas visando a realização da manutenção preventiva [4].

As técnicas de diagnóstico que são empregadas no monitoramento de danos estruturais nos dias atuais podem ser formuladas de duas formas:

a. Monitorar as mudanças da condição da estrutura e, se necessário, parar a operação do equipamento antes de piorar as condições;

b. Garantir que o dano iniciado não se estenda para uma situação de risco.

As técnicas de obtenção de (a) estão melhores definidas do que (b). 
Desta forma, a utilização de sensores inteligentes em conjunto com técnicas inteligentes para análise e tomada de decisões, se tornam possíveis alternativas para técnicas de monitoramento e diagnóstico de falhas.

Neste sentido, na literatura especializada encontram-se disponíveis diversos trabalhos que utilizam materiais e sistemas inteligentes para realizar o monitoramento e diagnóstico de falhas visando realizar manutenção preventiva que têm apresentado bom desempenho e precisão.

Existem trabalhos que contribuem de forma significativa, por exemplo, em [7] apresenta-se uma ferramenta baseada em redes neurais artificiais e transformada wavelet para realizar o diagnóstico de falhas em máquinas rotativas. No trabalho [10] os autores utilizaram uma rede multicamadas com treinamento via backpropagation para predição de falhas em vigas CFRP (reforçadas com fibra de carbono). Os autores em [8] combinaram a técnica de impedância elétrica com um algoritmo PSO (Particle Swarm Optimization) para diagnosticar falhas. Em [3] propõe-se um sistema fuzzy para detecção de falhas estruturais usando curvaturas das formas modais. No trabalho de [9] foi proposto um modelo utilizando transformada wavelet para avaliar integridade de estruturas de pontes através dos sinais de vibração. Em [11] foi proposto um algoritmo genético multinodal para diagnosticar danos em uma ponte de treliça de aço.

Neste artigo, apresenta-se um método para monitoramento e diagnóstico de falhas em estruturas mecânicas utilizando uma rede neural artificial ARTMAP-Fuzzy. A partir da aquisição e processamento dos sinais, aplica-se uma rede neural ARTMAP-Fuzzy [1] para identificar e caracterizar as falhas. Para avaliar este método, utilizou-se uma base de dados de sinais simulados a partir de um modelo numérico de uma viga de alumínio, que neste caso representa uma estrutura mecânica. A viga foi modelada através da técnica de elementos finitos e simulada em Matlab [6].

A escolha desta arquitetura (rede neural ARTMAP-Fuzzy), se deu pelo fato, de ser uma rede neural com arquitetura estável e plástica, o que garante uma vantagem em relação a outros tipos de redes neurais. A rede neural ARTMAP-Fuzzy permite a inclusão de um módulo de treinamento continuado, que possibilita a extração de conhecimento sem a necessidade de reiniciar o processo de treinamento quando novos padrões são apresentados, diferentemente do que ocorre com a maioria das redes neurais. Este recurso possibilita usar um conjunto reduzido de padrões na fase de treinamento, e a medida que forem realizadas as análises (aplicação), a extração do conhecimento é continuada, ou seja, trata-se de um sistema que busca o aperfeiçoamento com o passar o tempo.

Este artigo está organizado da seguinte forma: A descrição da rede ARTMAP-Fuzzy está na seção 2. Na seção 3 apresentam-se a modelagem e simulações realizadas. Na seção 4 apresenta-se a metodologia proposta. As aplicações e resultados são apresentados na seção 5 e por fim, a seção 6 apresenta a conclusão para este trabalho.

\section{Rede Neural ARTMAP-Fuzzy}

A rede neural artificial (RNA) ARTMAP-Fuzzy possui uma arquitetura baseada na teoria da ressonância adaptativa, isto é, pertence à família ART (Adaptive Resonance Theory) [2]. Esta rede corresponde a um sistema de aprendizado supervisionado composto por um par de módulos da teoria da ressonância adaptativa, $A R T_{a}-F u z z y$ e $A R T_{b}-F u z z y$, os quais recebem e classificam as entradas $\left(I_{a}\right)$ e saídas desejadas $\left(I_{b}\right)$ respectivamente, e o módulo de memória associativa inter- $A R T$ verifica o casamento das entradas e saídas com as categorias existentes. A figura 1 ilustra a arquitetura da rede neural ARTMAP-Fuzzy [1].

Os parâmetros fundamentais da rede neural ARTMAP-Fuzzy são [1]:

- Parâmetro de escolha $\alpha(\alpha>0)$ : Atua na seleção de categorias;

- Taxa de treinamento $\beta\left(\beta \in\left[\begin{array}{ll}0 & 1\end{array}\right]\right)$ : Controla a velocidade da adaptação da rede;

- Parâmetro de vigilância $\left(\rho_{a}, \rho_{b}\right.$ e $\left.\rho_{a b} \in\left[\begin{array}{ll}0 & 1\end{array}\right]\right)$ : Controla a ressonância da rede, isto é, o parâmetro responsável pelo número de categorias criadas. 


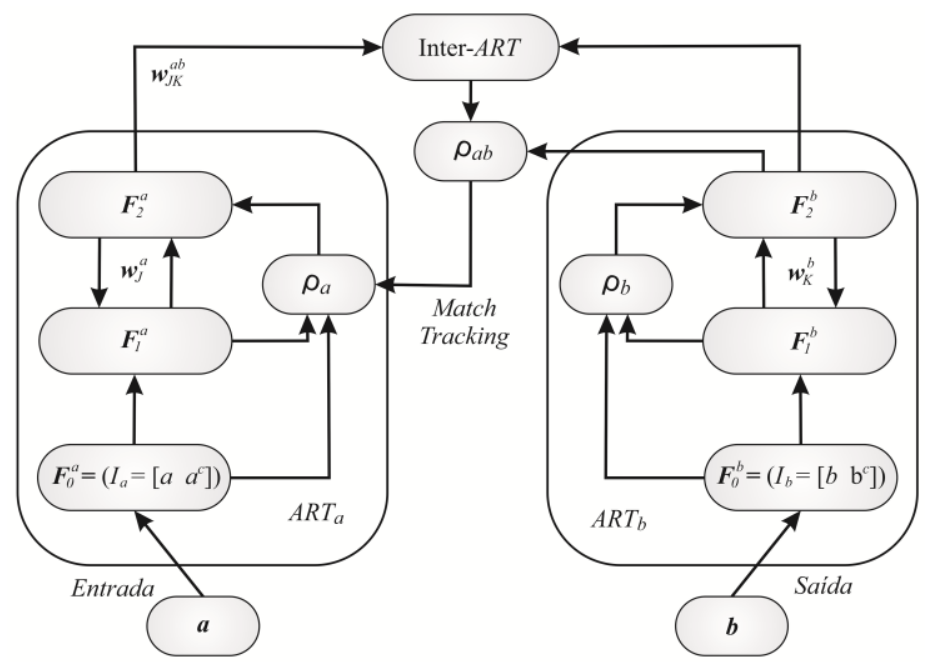

Figura 1: Estrutura da rede neural ARTMAP-Fuzzy [1].

Cada módulo ART é composto por três camadas: sendo $F_{0}$ a camada de entrada, $F_{1}$ a camada de comparação, e $F_{2}$ a camada de reconhecimento, que realiza o armazenamento em categorias. Os dados (I) fornecidos para a camada $F_{0}$ estão na forma de codificação de complemento, isto é, $I a=\left[\begin{array}{ll}a & a^{\mathrm{c}}\end{array}\right]$ e $I_{b}=\left[\begin{array}{ll}b & b^{\mathrm{c}}\end{array}\right]$. As camadas $F_{l}$ e $F_{2}$ são conectadas pelos pesos ( $w_{J}^{a}$ no $A R T a$ e $w_{K}^{b}$ no $A R T_{b}$ ). Estes pesos são utilizados no processo de escolha da categoria, sendo que para cada vetor de entrada $(I)$ em $F_{l}$ e o índice $J$ em $F_{2}$ é possível definir a função de escolha $T_{j}$ de acordo com (1), no qual $\left(^{\wedge}\right)$ é o operador fuzzy "and". A escolha da categoria é realizada conforme (2), sendo $J$ o índice ativo de $F_{2}[1]$.

$$
\begin{aligned}
& T_{j}=\frac{\left|I^{\wedge} w_{J}\right|}{\alpha+\left|w_{J}\right|} \\
& J=\arg \max T_{j}
\end{aligned}
$$

A ressonância ocorre caso o teste de vigilância (3) seja satisfeito para o índice ativo $J[1]$.

$$
\frac{\left|I^{\wedge} w_{J}\right|}{I} \geq \rho
$$

Caso o teste de vigilância (3) não seja satisfeito, ocorre o reset, e a função de escolha continua até que uma nova categoria $J$ satisfaça (3). Após o processo de ressonância nos módulos $A R T_{a}$ e $A R T_{b}$ deve-se verificar o casamento das categorias em ambos os módulos, esta etapa é realizada pelo módulo Inter- $A R T$.

O módulo Inter- $A R T$ verifica o casamento das informações entre os módulos $A R T$. Este processo é realizado pelo teste denominado Match Tracking descrito em (4), onde $y^{b}$ representa o vetor de atividade do módulo $A R T_{b}[1]$.

$$
\frac{\left|y^{b} \wedge w_{J K}^{a b}\right|}{y^{b}} \geq \rho_{a b}
$$

Caso o teste Match Tracking (4) não seja satisfeito, uma nova categoria do módulo $A R T_{a}$ deve ser escolhida e introduzida no processo de treinamento até que este critério seja satisfeito.

Após realizar os processos de ressonância para os módulos $A R T_{a}$ e $A R T_{b}$ e o Match Tracking para o módulo Inter- $A R T$ é realizada a adaptação dos pesos sinápticos $w_{J}^{a}, w_{K}^{b}$ e $w_{J K}^{a b}$ conforme (5), onde $J$ e $K$ são os índices ativos [1].

$$
\begin{aligned}
& w_{J}^{a+1}=\beta\left(I^{\wedge} w_{J}^{a}\right)+(1-\beta) w_{J}^{a} \\
& w_{J}^{b+1}=\beta\left(I^{\wedge} w_{K}^{b}\right)+(1-\beta) w_{K}^{b} \\
& w_{J K}^{a b+1}=\left\{\begin{array}{l}
1 \rightarrow J=j, K=k \\
0 \rightarrow J \neq j, K \neq k
\end{array}\right.
\end{aligned}
$$




\section{Modelagem e Simulação}

Para avaliar a metodologia proposta neste trabalho, foi utilizado o método de elementos finitos para modelar uma viga de alumínio na condição engastada-livre discretizada com 10 elementos finitos com 2 graus de liberdade cada. As propriedades do material empregado são: módulo de elasticidade $\mathrm{E}=700 \mathrm{GPa}$, densidade em massa $\gamma=2710 \mathrm{~kg} / \mathrm{m}^{3}$. As dimensões são: $500 \mathrm{~mm}$ de comprimento, $25 \mathrm{~mm}$ de largura e $5 \mathrm{~mm}$ de espessura. A figura 2 ilustra a viga modelada.

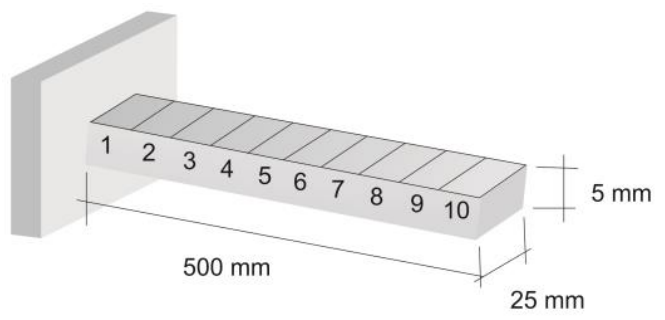

Figura 2: Viga modelada.

A partir do modelo de viga foram realizadas diversas simulações com diferentes porcentagens de desgastes e localizações das falhas. A base de dados gerada é constituída de sinais capturados por um acelerômetro fixado na viga. Em todas as simulações a viga foi excitada no $3^{\circ}$ grau de liberdade (elemento finito 2) e o sinal foi capturado no $19^{\circ}$ grau de liberdade (elemento finito 10). Sendo assim, foram simulados 540 sinais na estrutura, sendo 400 sinais sem desgaste (condição base-line) e 140 sinais com desgastes (falhas estruturais). Os sinais em falha foram simulados com níveis de desgaste de $1,5,10,15,20,25$ e 30\%. Para cada nível de desgaste a falha foi posicionada em dois locais, sendo os elementos finitos 3 e 5 . Na Tabela 1 apresenta-se a quantidade de simulações para cada nível de falha. Em cada nível de falha simulado foram simulados 10 sinais em cada localização, isto é, 10 falhas para cada nível no elemento finito 3 , e 10 falhas em cada nível para o elemento 5 .

Tabela 1 - Configurações das simulações.

\begin{tabular}{c|c}
\hline Nível de Falha & $\begin{array}{c}\text { Quantidade de } \\
\text { simulações }\end{array}$ \\
\hline $0 \%$ & 400 \\
$1 \%$ & 20 \\
$5 \%$ & 20 \\
$10 \%$ & 20 \\
$15 \%$ & 20 \\
$20 \%$ & 20 \\
$25 \%$ & 20 \\
$30 \%$ & 20 \\
\hline Total & 540 \\
\hline
\end{tabular}

\section{Metodologia Proposta}

O sistema de monitoramento e diagnóstico de falhas em estruturas mecânicas apresentado nesta seção é baseado em uma rede neural ARTMAP-Fuzzy [1]. O método proposto se divide em três módulos: aquisição de dados, treinamento da rede neural ARTMAP-Fuzzy e o diagnóstico dos dados. Na sequência apresenta-se o sistema de monitoramento e diagnóstico de falhas.

\subsection{Sistema de Monitoramento e Diagnóstico de Falhas Estruturais}

O sistema de monitoramento e diagnóstico de falhas proposto neste trabalho é composto por três módulos principais: a aquisição de dados, o treinamento e o diagnóstico da rede neural ARTMAP-Fuzzy.

O módulo de aquisição de dados é composto pelo aparato experimental para capturar os sinais na estrutura mecânica, como: sensores, atuadores, acelerômetros, etc. O conjunto de entrada da rede ARTMAP-Fuzzy é composto por sinais de resposta em frequência obtidos pelo módulo de aquisição de dados. 
O módulo de treinamento da rede neural ARTMAP-Fuzzy é realizado de forma off-line e serve para ajustar os pesos e criar as categorias (conhecimento) que serão utilizadas no diagnóstico dos dados. Neste módulo a rede é treinada com um conjunto de dados e será gerada uma quantidade de categorias igual ao número de sinais de entrada.

O módulo de diagnóstico é realizado de forma online. Neste módulo um novo conjunto de sinais diferente do conjunto de sinais utilizados na fase de treinamento deve ser apresentado a rede, e cada sinal será analisado individualmente. Todos os sinais são comparados com o conhecimento (categorias criadas na fase de treinamento) obtido pela rede neural ARTMAPFuzzy e assim, quando houver um casamento entre os padrões classifica-se em categoria o sinal apresentado a rede ARTMAP-Fuzzy. Obtida a categoria correspondente a um determinado padrão é, portanto, identificada a intensidade (porcentagem de falha) e a localização da falha na estrutura. O fluxograma do funcionamento do sistema de monitoramento e diagnóstico de falhas é apresentado na figura 3.

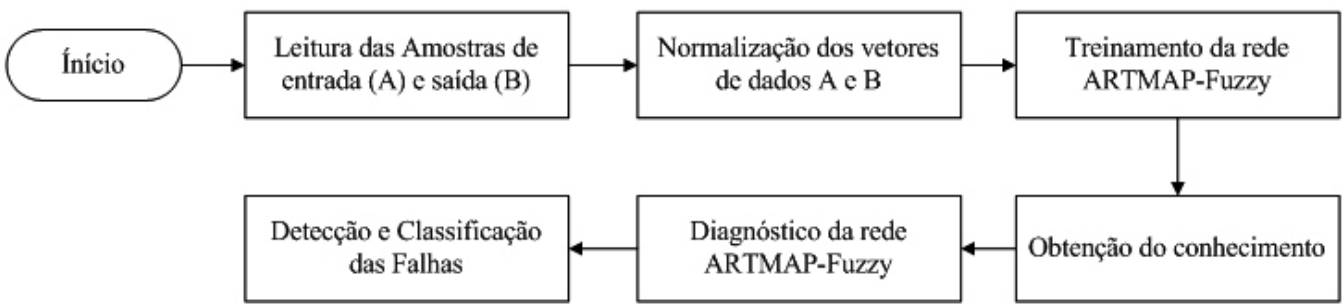

Figura 3: Funcionamento do Sistema de Diagnóstico de Falhas.

\section{Aplicações e Resultados}

Nesta seção apresentam-se os resultados obtidos com a aplicação da metodologia proposta na base de dados simulada. Todos os testes foram realizados utilizando um PC Intel Core 2 Duo $1.9 \mathrm{GHz}, 2$ GB de Memória RAM, e sistema operacional Windows 7 Ultimate 32 bits. O algoritmo foi desenvolvido em MATLAB [6].

Para avaliar a metodologia proposta analisou-se a eficiência, precisão e o tempo computacional no processo de diagnóstico de falhas em estruturas mecânicas.

Os parâmetros da rede neural ARTMAP-Fuzzy utilizados no processo de treinamento e diagnóstico foram: $\alpha=0,1, \beta=1, \rho_{a}=0,8, \rho_{b}=1$ e $\rho_{a b}=1$.

$\mathrm{Na}$ fase de treinamento foi utilizado um conjunto de dados com 398 sinais, sendo 300 sinais da estrutura em condições normais (base-line) e 98 sinais da estrutura em falha. Este conjunto de dados corresponde a $70 \%$ dos dados disponíveis. Para avaliar a rede neural ARTMAP-Fuzzy na fase de diagnóstico foi utilizado um conjunto de dados com 142 sinais, sendo 100 sinais da estrutura sem falha e 42 sinais da estrutura em falha. Estes dados utilizados nos testes representam $30 \%$ dos dados disponíveis. A base de dados simulada conta com 14 padrões diferentes de falhas.

$\mathrm{Na}$ Tabela 2 apresentam-se os resultados obtidos pelo sistema de diagnóstico de falhas em estruturas quando aplicado aos dados da viga de alumínio.

Tabela 2 - Resultados obtidos pelo método.

\begin{tabular}{c|c|c}
\cline { 2 - 3 } & Fase de Treinamento & Fase de Diagnóstico \\
\hline $\begin{array}{c}\text { Amostras } \\
\text { Utilizadas }\end{array}$ & 398 & 142 \\
\hline $\begin{array}{c}\text { Classificações } \\
\text { Corretas }\end{array}$ & 398 & 142 \\
\hline $\begin{array}{c}\text { Classificações } \\
\text { Erradas }\end{array}$ & 0 & 0 \\
\hline Acerto $(\%)$ & 100,00 & 100,00 \\
\hline Tempo $(m \mathrm{~s})$ & 645,1 & 139,00 \\
\hline
\end{tabular}

Para obtenção destes resultados o sistema passou por uma fase experimental de testes e ajustes de parâmetros. Os resultados apresentados foram obtidos com a melhor configuração da 
rede neural ARTMAP-Fuzzy. O resultado foi obtido através de um teste de referência cruzada, em que o sistema foi executado 20 vezes a fim de garantir a veracidade dos resultados. Foi possível observar que o sistema de diagnóstico de falhas em estruturas apresenta um bom desempenho, com um índice de acerto igual a $100 \%$. Ao utilizar uma rede neural ARTMAPFuzzy, sugere-se utilizar 70\% dos dados para o treinamento e 30\% para o diagnóstico. Quanto mais conhecimento obtido no treinamento, mais eficiente é o processo de diagnóstico.

\section{Conclusão}

O sistema de diagnóstico de falhas em estruturas mecânicas proposto neste artigo apresentou excelentes resultados, obtendo um índice de acerto de $100 \%$ para a melhor configuração da rede neural ARTMAP-Fuzzy. A fase de treinamento é a que demanda maior tempo computacional, no entanto é executada de forma off-line não acarretando prejuízo ao sistema. A fase de diagnóstico do sistema, a partir da aquisição dos sinais, é realizada rapidamente, com tempo inferior a 130 milésimos de segundos para cada sinal, o que proporciona rapidez na tomada de decisão em tempo real. Desta forma, conclui-se que o sistema de diagnóstico proposto com base em uma rede neural ARTMAP-Fuzzy é bastante eficiente, confiável, robusto e preciso para realizar diagnóstico de falhas, visando a manutenção preventiva de estruturas mecânicas.

\section{Agradecimentos}

Os autores agradecem a CAPES e CNPQ (proc. $n^{\circ} 301769 / 2012-5$ ) pelo apoio financeiro de pesquisa.

\section{Referências}

[1] G. A. Carpenter; S. Grossberg, N. Markuzon; J. H. Reynold and D. B. Rosen. Fuzzy ARTMAP: A neural network for incremental supervised learning of analog multidimensional maps. IEEE Transactions on Neural Network, vol. 3, n. 5, p. 689-713, (1992).

[2] G. A. Carpenter and S. Grossberg. A massively parallel architecture for a self-organizing neural pattern recognition machine. Computer Vision, Graphics, and Image Processing, p. 54-115, (1987).

[3] M. Chandrashekhar; R. Ganguli. Structural damage detection using modal curvature and fuzzy logic. Structural Health Monitoring, USA, v. 8, n. 4, p. 267-282, (2009).

[4] V. R. Franco; D. D. Bueno; M. J. Brennan; A. A. Cavalini JR.; C. G. Gonsalez; V. Lopes JR. Experimental damage location in smart structures using Lamb waves approaches. In: Brazilian Conference on Dynamics, Control and Their Applications - DINCON. p. 1-4. (2009).

[5] S. R. Hall. The effective management and use of structural health data. In: International Workshop on Structural Health Monitoring, p. 265-275. (1999).

[6] Matlab (2011). 7.8 Version, Mathworks Company.

[7] D. F. A. Santiago. Diagnóstico de falhas em máquinas rotativas utilizando transformada de wavelet e redes neurais artificiais. Campinas, 2004. 117p. Tese (Doutorado em Engenharia Mecânica) - Faculdade de Engenharia, Universidade Estadual de Campinas.

[8] A. Tebaldi; L. S. COELHO; V. LOPES JR. Detecção de falhas em estruturas inteligentes usando otimização por nuvem de partículas: fundamentos e estudo de casos. Revista Controle \& Automação, Brasil, v.17, n. 3, p. 312-330, 2006.

[9] C. Xiang-Jun; G. Zhan-Feng; G. Qiang. Application of wavelet analysis in vibration signal processing of bridge structure. International Conference on Measuring Technology and Mechatronics Automation. p. 671-674, (2010).

[10] S. Zheng; X. Wang; L. Liu. Damage detection in composite materials based upon the computational mechanics and neural networks. In: European Workshop on Structural Health Monitoring, Munich, p. 609-615 (2004).

[11] F. L. Wang; T. H. T. Chan; D. P. Thambiratnam and A. C. C. Tan. Damage Diagnosis for Complex Steel Truss Bridges Using Multi-Layer Genetic Algorithm. Journal of Civil structural Health Monitoring, Springer-Verlag, p. 117-217, (2013). 